\title{
Plausible explanations for the variations of orbital period in the old nova DQ Herculis ${ }^{\star}$
}

\author{
Z. B. Dai ${ }^{1,2,3}$ and S. B. Qian ${ }^{1,2}$ \\ 1 National Astronomical Observatories/Yunnan Observatory, Chinese Academy of Sciences, PO Box 110, 650011 Kunming, \\ PR China \\ e-mail: zhibin_dai@ynao.ac.cn \\ 2 United Laboratory of Optical Astronomy, Chinese Academy of Science (ULOAC), 100012 Beijing, PR China \\ 3 Graduate School of the CAS, Beijing, PR China
}

Received 4 September 2008 / Accepted 28 April 2009

ABSTRACT

\begin{abstract}
Aims. Several mechanisms are presented to explain the observed small variation in the orbital period of the old nova DQ Herculis. Methods. We have combined two new CCD times of light minimum of DQ Herculis with all 226 available times of light minimum, including 79 visual observations, for the new orbital period analysis.

Results. Based on this analysis, the best-fit of the O-C diagram for DQ Herculis is a quadratic-plus-sinusoidal fit. A secular orbital period increase with a rate of $9.5( \pm 0.1) \times 10^{-12} \mathrm{~s} \mathrm{~s}^{-1}$ is confirmed, which corresponds to a lower limit of the mass transfer rate of $7.2( \pm 3.2) \times 10^{-9} M_{\odot} \mathrm{yr}^{-1}$. We investigate three plausible mechanisms (direct change of the red dwarf's radius, Applegate's mechanism and the light travel-time effect) to explain the quasi-periodic variation shown in the $\mathrm{O}-\mathrm{C}$ diagram. Although previous works have suggested that solar-type magnetic cycles in the red dwarf can explain the quasi-periodic variation in the orbital period, we were not able to reproduce this finding. Accordingly, a light trave-time effect is proposed, with a brown dwarf as a tertiary component with a significance level of $\gtrsim 77.8 \%$ orbiting around nova DQ Herculis. In order to interpret the small departure from the best-fit near 60000 cycles, we assume an eccentric orbit of the third body with a small eccentricity. However, a satisfying result was obtained because the eccentricity $e=0.12$ is close to zero. The parameters of this elliptical orbit are similar to that of a circular orbit.
\end{abstract}

Key words. stars: novae, cataclysmic variables - stars: binaries: eclipsing - stars: individual: DQ Herculis

\section{Introduction}

The remnant of the nova Herculis 1934, named DQ Herculis, was first detected at a magnitude of 3.3 (Campbell 1935). It is not only a well-observed prototype of intermediate Polar stars, but also a moderately slow galactic nova. This means that the binary system not only contains a white dwarf with a strong magnetic field, which can partially control the accreting stream from the red dwarf along the magnetic field lines to fall onto one or both magnetic poles of the white dwarf (Patterson 1994), but also has thermonuclear runaway on the surface of the white dwarf (Starrfield 1989). The discovery of eclipses in DQ Herculis (Walker 1956) was pivotal in establishing the standard picture of cataclysmic variables. At the same time, Walker (1956) reported a coherent $71 \mathrm{~s}$ photometric oscillation. The spectral type of the red dwarf is estimated to be M3 due to the detection of faint TiO bands (Young \& Schneider 1981). Since DQ Herculis is an eclipsing binary with an orbital period of $4^{\mathrm{h}} 39^{\mathrm{m}}$ and has been extensively observed, its properties are well known. For example, recent estimations of $M_{1}=0.6 M_{\odot}$ and $M_{2}=0.4 M_{\odot}$ (Horne et al. 1993) correct the previous results from earlier works (e.g. Robinson 1976; Smak 1980).

Since the mass transfer in DQ Herculis detected by Kraft (1959), a variation in the orbital period is expected. Mumford (1969) and Nather \& Warner (1969) suggested that the orbital period is increasing, and later works (Patterson et al. 1978; Africano \& Olson 1981; Zhang et al. 1995; Wood et al. 2005)

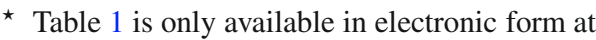
http://www . aanda. org indicated that a sinusoidal modulation with a period of $\sim 14 \mathrm{yr}$ is likely. However, an updated O-C analysis (Wood et al. 2005) concluded that there is not strong evidence for a secular orbital period increase. They did not produce an explanation for the cyclical variation in the $\mathrm{O}-\mathrm{C}$ diagram of DQ Herculis. According to Patterson et al. (1978), light travel-time effects cannot be applied to explain the $\mathrm{O}-\mathrm{C}$ cyclic variation because a similar variation is not present in the $71 \mathrm{~s}$ oscillation timings. Therefore, there is no convincing explanation for the orbital period variation of DQ Herculis so far.

226 available times of light minimum from 1954 to 2008 are presented in Sect. 2, including two new data points from our observations and 79 visual data points. Section 3 gives the $\mathrm{O}-\mathrm{C}$ analysis for DQ Herculis. A discussion of the possible mechanisms for orbital period change is given in Sect. 4 .

\section{Observation of times of light minimum}

Two new times of light minimum are obtained from our CCD photometric observations with the PI1024 TKB CCD photometric system attached to the 1.0-m reflecting telescope at the Yunnan Observatory in China. The first CCD photometric observation of DQ Herculis was carried out on February 10, 2008 in white light, and the second observation on March 28, 2008 was through the $R$-filter, which is close to the Johnson standard photometric system. Two nearby stars that have a similar brightness in the same viewing field of the telescope were chosen as the comparison star and the check star. All images were reduced by using PHOT (measured magnitudes for a list of stars) in the aperture photometry package of IRAF. Two CCD times of light 


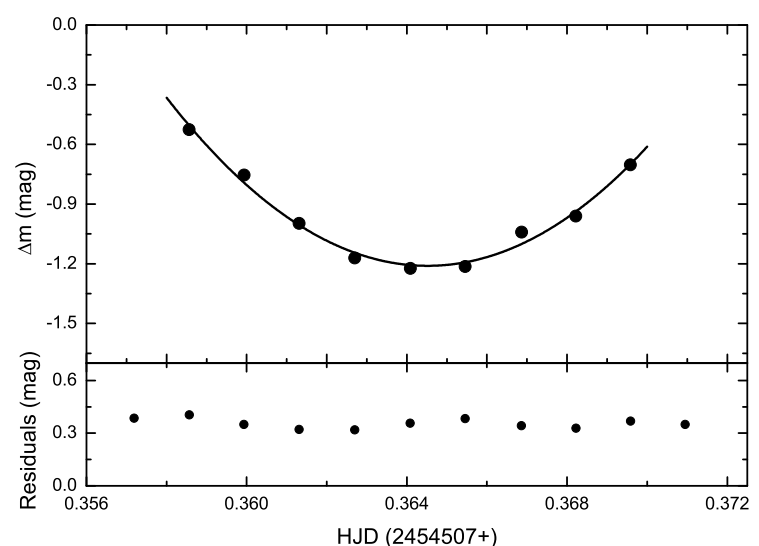

Fig. 1. The light curve of the eclipsing part of DQ Herculis shown in the upper figure, obtained on Feb. 10, 2008 in white light. The solid line represents the best parabolic fit. The lower figure shows the residuals of differential photometry.

minimum were derived by using a parabolic fitting method. Both light curves of eclipsing parts and their parabolic fits are displayed in upper planes of Figs. 1 and 2, respectively. The residuals of differential photometries shown in the lower planes of Figs. 1 and 2 suggest that the times of light minimum in our observations can be derived by a parabolic fit with high precision.

As the two new eclipsing times obtained from CCD observations are to 4 significant figures, we infer that the previous times of light minimum obtained by using photographic or visual methods without given error, should have lower accuracy. Therefore, we assume that the CCD or photoelectric data and the photographic or visual data without given errors have default errors of 0.0001 and 0.001 , respectively. The collected times of light minimum were checked again because some data were obtained from many years ago, and some identical times of light minimum may have small differences, as they were observed at different observatories or in different filters. In this case, the average value should be reliable and may be applied. In addition, Zhang et al. (1995) and Wood et al. (2005) reported their 44 eclipse times in Heliocentric Julian Ephemeris Dates (HJED), which correspond to ephemeris time (ET). Thus, we converted them to Heliocentric Julian Dates (HJD), which correspond to coordinated Universal time (UTC). All 226 available times of light minimum covering half a century listed in Table 1 have been checked carefully, and those obviously inaccurate were corrected or abandoned. For example, the 7 photographic data points before 1954 are not used in this paper because of the very large scatter.

\section{Analysis of orbital period changes}

The ephemeris derived by Africano \& Olson (1981) is used to calculate the $\mathrm{O}-\mathrm{C}$ values of DQ Herculis and, after linear revision, the new epochs and average orbital period of DQ Herculis were derived as

$T_{\min }=$ HJD $2434954.94306(2)+0 \mathrm{~d} \cdot 1936209219(3) E$,

with variance $\sigma_{1}=1.42 \times 10^{-3}$. The $\mathrm{O}-\mathrm{C}$ values versus epoch numbers are plotted in Fig. 3 which suggests a trend of cyclic variation. Although Wood et al. (2005) pointed out that a sinusoidal fit is formally significant but not compelling, the two new eclipsing times we obtained could alter this conclusion because the O-C values since 1985 cannot be fitted by a straight line. Moreover, we suggested that the most important cause of the

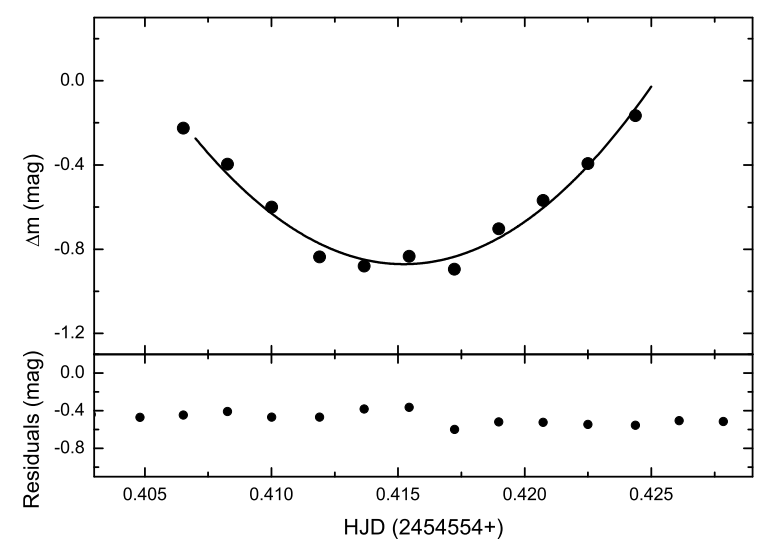

Fig. 2. Upper panel is the $R$-band light curve of the eclipsing part of DQ Herculis obtained on Mar. 28, 2008. The solid line represents the best parabolic fit. The lower figure shows the residuals of differential photometry.

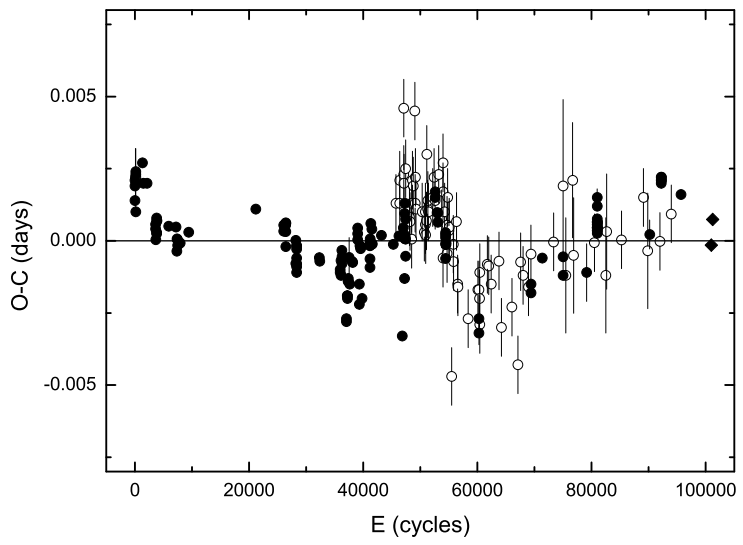

Fig. 3. The O-C values calculated with Eq. (1) versus the cycles. The 79 visual data points and the photoelectric or CCD data are plotted as open circles and solid circles, respectively.

non-compelling sine fit is the neglect of a quadric term in the $\mathrm{O}-\mathrm{C}$ analysis. Accordingly, based on all 226 data points, a leastsquare solution of the quadric ephemeris leads to

$$
\begin{aligned}
(\mathrm{O}-\mathrm{C})_{1}= & 0.00112( \pm 0.00002)-7^{\mathrm{d}} .6( \pm 0.1) \times 10^{-8} E \\
& +8.4( \pm 0.1) \times 10^{-13} E^{2},
\end{aligned}
$$

with variance $\sigma_{2 q}=1.38 \times 10^{-3}$. This quadric fit and its residuals (i.e. $\left.(\mathrm{O}-\mathrm{C})_{2}\right)$ are presented in the top and middle planes of Fig. 4 , respectively. The $\mathrm{O}-\mathrm{C}$ diagram, after removing the quadric term shown in middle planes of Fig. 4, also shows a cyclical period change. A simple sinusoidal function is used to fit the $(\mathrm{O}-\mathrm{C})_{2}$ and a least-square solution yields

$$
\begin{aligned}
(\mathrm{O}-\mathrm{C})_{2}= & 3^{\mathrm{d}} .1\left( \pm 0^{\mathrm{d}} 1\right) \times 10^{-4} \sin \left[0.011^{\circ}\left( \pm 0.0002^{\circ}\right) E\right. \\
& \left.+90.0^{\circ}\left( \pm 3.4^{\circ}\right)\right],
\end{aligned}
$$

with variance $\sigma_{2 s}=1.25 \times 10^{-3}$. Using the statistic parameter $\lambda$ of the $F$-test as defined by Pringle (1975), we obtained $F(1,223)=13.1$, which corresponds to $a>99.99 \%$ confidence level for the existence of quadric term. However, since the simple sine fit fails the $F$-test, we performed a regular $\chi^{2}$-test to measure the goodness-of-fit of Eq. (3). The calculated parameter $\chi^{2}=39.9$ indicates that it is a significant fit. Since the simple fit cannot simultaneously adjust the quadric term and the sinusoidal term in the $\mathrm{O}-\mathrm{C}$ diagram to search for a best fit, in order to further reduce the residuals we attempted to use a simultaneous quadratic-plus-sinusoidal formula to fit this variation. The 


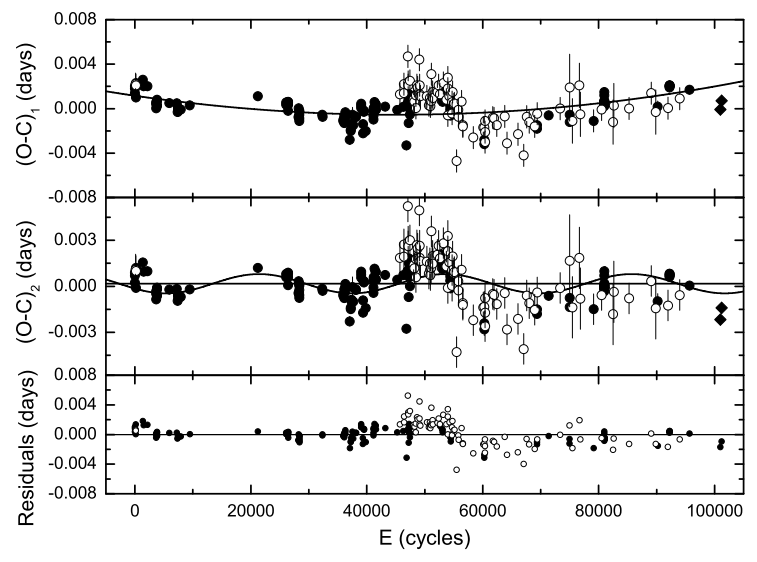

Fig. 4. The O-C values of DQ Herculis fitted with the formulas displayed in Eqs. (2) and (3). The fitting curves are plotted in the top and middle figure, respectively. The residuals and their linear fitted solid line are presented in the bottom plane.

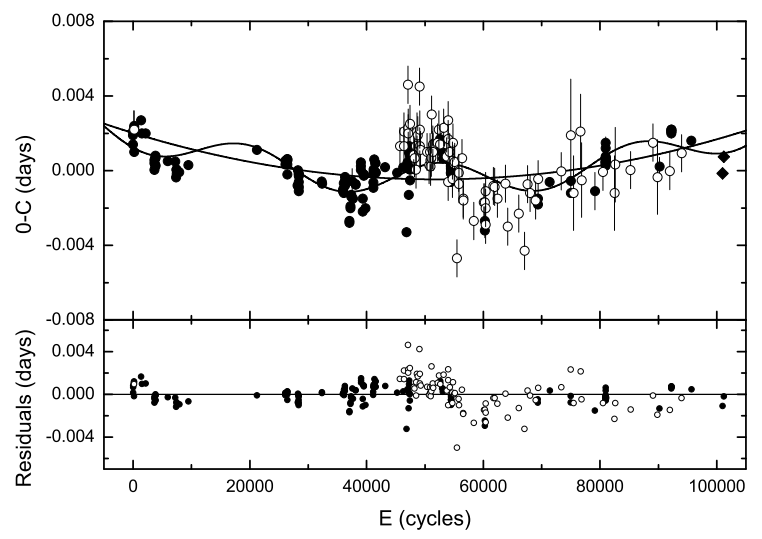

Fig. 5. The O-C values of DQ Herculis obtained from the elements in Eq. (4). The fitting curves are plotted in the top figure. The residuals are presented in the bottom plane.

least-square solution leads to

$$
\begin{aligned}
\mathrm{O}-\mathrm{C}= & 2.01( \pm 0.03) \times 10^{-3}-9^{\mathrm{d}} .6( \pm 0.1) \times 10^{-8} E \\
& +9^{\mathrm{d}} .2\left( \pm 0^{\mathrm{d}} .1\right) \times 10^{-13} E^{2}+8^{\mathrm{d}} .9( \pm 0.2) \times 10^{-4} \\
& \times \sin \left[0.0108^{\circ}\left( \pm 0.0002^{\circ}\right) E+60.9^{\circ}\left( \pm 0.9^{\circ}\right)\right]
\end{aligned}
$$

with variance $\sigma_{3}=1.17 \times 10^{-3}$. The residuals are displayed in the bottom panel of Fig. 5. The $F$-test to assess the significance of the quadratic and sinusoidal terms in Eq. (4) is valid, as long as the parameter $\lambda$ is corrected to be

$\lambda=\frac{\left(\sigma_{1}^{2}-\sigma_{3}^{2}\right) / 4}{\sigma_{3}^{2} /(n-6)}$,

where $n$ is the number of data points. Thus, a calculation gives $F(4,220)=26.0$, which indicates that it is significant well above the $99.99 \%$ level. Therefore, both separate and simultaneous fits suggest that the best-fit of the O-C diagram for DQ Herculis should be the $\sim 17.7( \pm 0.3)$ yr quasi-periodic modulation shown in the top and middle panels of Fig. 5 superimposed on a secular orbital period increase. The variational period $\sim 17.7( \pm 0.3) \mathrm{yr}$ is in agreement with the result of $\sim 14 \mathrm{yr}$ from earlier works. The sine curve shown in the top panel of Fig. 5 does not well describe the visual data of 60000 cycles observed in 1985, as is also shown in Wood et al. (2005). Although this poor fit is caused mainly by the visual data, two photoelectric observations at cycles 60281 and 60286 , also present a large departure from the fit, as the visual data do. This means that the visual data may show a real episode of variation of the eclipse minima. Therefore, the variation in the $\mathrm{O}-\mathrm{C}$ diagram should be quasiperiodic, as discussed in Sect. 4.

Although Pringle (1975), Africano \& Olson (1981) and Wood et al. (2005) suggested that the orbital period of DQ Herculis is constant, the residuals of this quadratic-plussinusoidal fit shown in the bottom panel of Fig. 5 never show a detectable variation, which implies the existence of quadratic variation in $\mathrm{O}-\mathrm{C}$ diagram. Moreover, the standard model of cataclysmic variables (Warner \& Nather 1971; Smak 1971) indicates that mass transfer is a common phenomena. According to the coefficient of the quadratic term in Eq. (4), the orbital period change rate of DQ Herculis, $\dot{P}$, can be calculated to be $9.5( \pm 0.1) \times 10^{-12} \mathrm{~s} \mathrm{~s}^{-1}$.

\section{Discussion}

\subsection{Secular orbital period increase}

Considering that the secular orbital period change for DQ Herculis remains a matter of debate, the mass transfer between both components may be low or non-existent, a situation that corresponds to the presence of a faint hot spot in many eclipse light curves of DQ Herculis (Bianchini et al. 2004). Although the accurate masses of both components are still not available, it is definite that the mass ratio, $q=M_{2} / M_{1}$, is less than unity. Therefore, mass transfer from the red dwarf to the white dwarf is a very reasonable explanation for the orbital period increase shown in Figs. 4 and 5. According to Kepler's third law, the orbital period change with conservation can be given by

$\frac{\dot{P}}{P}=3 \frac{M_{2}-M_{1}}{M_{1}} \frac{\dot{M}_{2}}{M_{2}}$

where $\dot{M}_{2}$ is mass transfer rate from the red dwarf. Using the masses of both components $\left(M_{1}=0.6( \pm 0.07) M_{\odot}\right.$ and $M_{2}=$ 0.4( \pm 0.05$\left.) M_{\odot}\right)$ derived by Horne et al. (1993), a lower limit of the mass transfer rate, $\dot{M}_{2}=-7.2( \pm 3.2) \times 10^{-9} M_{\odot} \mathrm{yr}^{-1}$ is obtained, which is the same order of magnitude as that derived by Zhang et al. (1995), and should be considered as a lower limit. Thus, if there are extra mechanisms of mass and angular momentum loss in DQ Herculis, then a considerable mass transfer rate from the red dwarf to the white dwarf is needed to produce the observed orbital period increase.

\subsection{Quasi-periodic variation}

\subsubsection{Variations of the red dwarf's radius}

$\mathrm{A} \sim 17.7( \pm 0.3)$ yr quasi-period variations is seen in the $\mathrm{O}-\mathrm{C}$ diagram of DQ Herculis. Warner (1988) applied a technique sensitive to mass overflow, which means that the mass transfer from the red dwarf can be changed by a slight variation of its radius. This can explain the cyclical change in the $\mathrm{O}-\mathrm{C}$ diagram. He proposed that a change in radius of the tidally distorted secondary star is the result of solar-type magnetic cycles. The variation of radius of the red dwarf, $\Delta R$, can be described as,

$\frac{\Delta R}{R}=A \sin (\Omega t)$

where $A$ is the amplitude of radius variations. The modulation of mass transfer rate $\dot{M}$ caused by radius variation is given by

$\dot{M}=\dot{M}_{0} \mathrm{e}^{3.1 \times 10^{3} \frac{\Delta R}{R}}$, 
where $\dot{M}_{0}$ is the rate for the undisturbed state (i.e. $\Delta R=0$ ) and the times of eclipse minima will vary as (Warner 1988)

$$
(\mathrm{O}-\mathrm{C})_{\text {orbital }}=13.0 k_{2} q^{2 / 3}(1+q)^{-5 / 3} \frac{A}{\Omega} \cos (\Omega t),
$$

where $t$ is in days, $k_{2}$ and $q$ are the apsidal coefficient and the mass ratio, respectively. Compared to Eq. (4), (O-C) orbital should be its sinusoidal part. Thus, $\Omega$ is found to be $9.74 \times 10^{-4} \mathrm{~d}^{-1}$. Horne et al. (1993) obtained the mass ratio $q=0.62 \pm 0.05$ for DQ Herculis. The apsidal coefficient $k_{2}$ is 0.143 for a $M \simeq$ $0.4 M_{\odot}$ star (Warner 1988). Therefore, the amplitude of $\Delta R / R$ can be calculated to be $A=1.43( \pm 0.03) \times 10^{-6}$, which is similar to the result of Warner (1988). However, Marsh \& Pringle (1990) found that Warner (1988) did not consider whether enough energy is available to produce such changes on the observed time scales. Therefore, we attempted to estimate the time scale of change, $t_{\mathrm{ch}}$, for DQ Herculis, which can be given by the equation (Marsh \& Pringle 1990)

$t_{\mathrm{ch}}>2 \times 10^{7} f \frac{\Delta R}{R}\left(\frac{M_{2}^{2}}{L_{2} R_{2}}\right) \mathrm{yr}$,

where $f, M_{2}$ and $L_{2}$ are outer mass fraction, the mass and the luminosity of the red dwarf, respectively. Schneider \& Greenstein (1979) estimated the spectral type of the secondary star of DQ Herculis to be M4 or later, and Young \& Schneider (1981) pointed out that its spectral type should be $M 3^{+} V$ due to the TiO band ratio found. This means that the luminosity of the red dwarf can be estimated to be $1.07 \times 10^{32} \mathrm{erg} \mathrm{s}^{-1}$, and the red dwarf may be a fully convective star. Thus, Eq. (10) becomes

$t_{\mathrm{ch}}>3.38 \times 10^{2} f \mathrm{yr}$,

with $R_{2}=0.49 R_{\odot}$ (Horne et al. 1993). If the observed period change is caused by a change in the mean radius of the red dwarf, then the $\sim 17.7( \pm 0.3)$ yr quasi-periodic oscillation shown in Fig. 5 indicates $f \sim 0.052$, which means that the radius change only involves an extremely thin shell (i.e. $M_{\text {shell }} \sim 0.02 M_{\odot}$ ). This is obviously unreasonable for $a \simeq 0.4 M_{\odot}$ fully convective red dwarf star. Thus, a change in the size of the red dwarf of DQ Herculis requires too much energy. This further supports the conclusions of Hall (1991) and Marsh \& Pringle (1990).

\subsubsection{Applegate's mechanism}

Since Marsh \& Pringle (1990) pointed out the required energy problem, Applegate (1992) proposed an alterative mechanism, which is magnetic activity-driven change in the quadrupole momentum of the red dwarf. According to this mechanism, the relationship between the orbital period $P$ and the variation of the quadrupole momentum $\Delta Q$ is given by

$\frac{\Delta P}{P}=-9\left(\frac{R_{2}}{a}\right)^{2} \frac{\Delta Q}{M_{2} R_{2}^{2}}$,

where $R_{2}$ and $a$ are the radius of the secondary star and the binary separation, respectively. Using $a=1.41( \pm 0.05) R_{\odot}$ and $M_{2}=$ $0.40( \pm 0.05) M_{\odot}$ (Horne et al. 1993), $\Delta Q$ can be calculated to be $-7.4( \pm 1.1) \times 10^{40} \mathrm{~g} \mathrm{~cm}^{2}$.

In order to discuss the internal angular momentum transport, which can produce the orbital period variation in a fully convective star, the whole star is split into an inner core and an outer shell. The energy $\Delta E$ required to cause the angular momentum

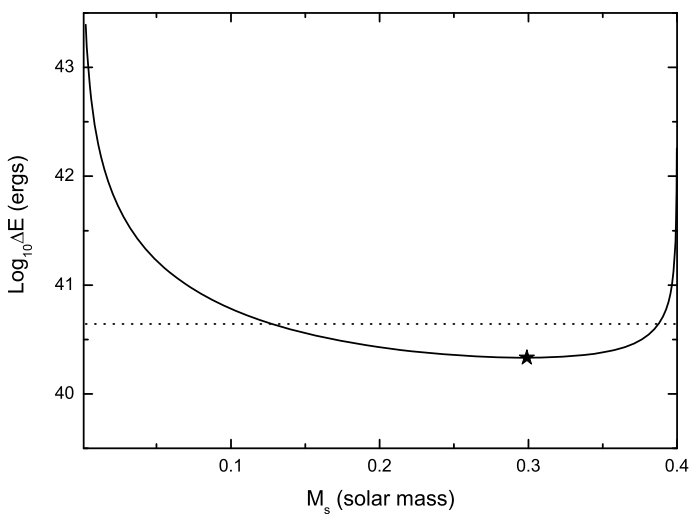

Fig. 6. The correlation between the energy required to produce the orbital period oscillation of DQ Herculis by using the Applegate (1992) mechanism and the assumed different shell masses of the fully convective red dwarf (solid line). The star denotes the minimum required energy, and the dotted line is the total radiant energy of the red dwarf over the whole oscillation period.

transfer $\Delta J$ between the shell and core, is estimated by the formula (Applegate 1992)

$\Delta E=\Omega_{\mathrm{dr}} \Delta J+\frac{(\Delta J)^{2}}{2 I_{\mathrm{eff}}}$

where $\Omega_{\mathrm{dr}}=\Omega_{\text {shell }}-\Omega_{\text {core }}$ is the initial differential rotation, and $I_{\text {eff }}=I_{\text {shell }} I_{\text {core }} /\left(I_{\text {shell }}+I_{\text {core }}\right)$ is the reduced rotational inertia. The required energy $\Delta E$ versus each different shell mass $M_{\mathrm{S}}$ is plotted in Fig. 6. The lowest required energy $\Delta E_{\min }$ is $2.15 \times 10^{40} \mathrm{erg}$ at $M_{\mathrm{s}} \simeq 0.30 M_{\odot}$. Assuming that the spectral type of the red dwarf is $\mathrm{M} 3 \mathrm{~V}$, the total radiant energy $E_{0}$ in a complete variation period of $17.7 \mathrm{yr}$, which is the maximum energy offered by the luminosity variations of the M3V-type red dwarf, can be estimated to be $5.95 \times 10^{40} \mathrm{erg}$. The apparent magnitude variation of DQ Herculis is $\Delta m_{\mathrm{v}} \simeq 0.6$ (Warner 1988), which gives the energy emitted by the red dwarf of $E_{0} \simeq 4.39 \times 10^{40} \mathrm{erg}$. Since $E_{0}$ of is the same order of magnitude as $\Delta E_{\min }$, we consider that Applegate's mechanism may have difficulty in explaining the observed oscillation in orbital period (i.e. $E_{0} / \Delta E_{\min } \sim 2$ ).

\subsubsection{Light travel-time effect}

Another plausible mechanism to explain the sinusoidal variation in the $\mathrm{O}-\mathrm{C}$ diagram is the light travel-time effect, which is caused by a perturbations from a tertiary component (Irwin 1952; Borkovits \& Hegedues 1996). Patterson et al. (1978) suggested that the light travel-time effect is not a good explanation for the periodic oscillation in the $\mathrm{O}-\mathrm{C}$ diagram of DQ Herculis because no evidence for a similar sinusoidal variation is found in the O-C diagram of $71 \mathrm{~s}$. However, the detection of $71 \mathrm{~s}$ has a large error because of the very short time scale and the large oscillation phase shifts, which may affect the continuity of the times of maximum and the precision of the ephemeris. Moreover, there is no clear evidence that the $\mathrm{O}-\mathrm{C}$ diagrams of $71 \mathrm{~s}$ well reflect the motion of the mass-center of DQ Herculis. Therefore, we investigated the possible light travel-time effect.

The strictly sinusoidal fit presented in Fig. 5 is a special case that corresponds to the assumption of a circular orbit. We assumed that the small departure near 60000 cycles shown in the top panel of Fig. 5 is caused by a small orbital eccentricity of the third body, which can be described by (Irwin 1952);

$\mathrm{O}-\mathrm{C}=\frac{a \sin i}{c}\left[\frac{1-e^{2}}{1+e \cos v} \sin (v+\omega)+e \sin \omega\right]$, 
Table 2. The best-fit coefficients.

\begin{tabular}{ccc}
\hline \hline & Coefficients & Errors \\
\hline$a_{0}$ & 0.00201 & 0.00002 \\
$\alpha$ & $-9.6 \times 10^{-8}$ & $0.1 \times 10^{-8}$ \\
$\beta$ & $9.3 \times 10^{-13}$ & $0.1 \times 10^{-13}$ \\
$a_{1}$ & -0.00084 & 0.00002 \\
$b_{1}$ & -0.00037 & 0.00001 \\
$a_{2}$ & -0.00003 & 0.00001 \\
$b_{2}$ & -0.00004 & 0.00001 \\
\hline
\end{tabular}

where $a, c, i, e, v$ and $\omega$ are the semi-major axis of elliptic orbit, light speed, orbital inclination, eccentricity, true anomaly and the longitude of periastron from the ascending node. If the assumed orbital eccentricity is small enough (i.e. $e<0.6$ ), then Eq. (9) can be replaced with a simple approximate formula (Kopal 1959, 1978),

$$
\begin{aligned}
\mathrm{O}-\mathrm{C}= & a_{0}+a_{1} \cos (\Omega E)+b_{1} \sin (\Omega E)+a_{2} \cos (2 \Omega E) \\
& +b_{2} \sin (2 \Omega E),
\end{aligned}
$$

where $\Omega$ is the angular frequency of elliptic orbit, which corresponds to the orbital period of the third body, $P_{3} . a \sin i$ is the projected distance from the binary pair to the mass-center of this triple system. $a \sin i$, e and $\omega$ can be given by

$a \sin i=c \sqrt{a_{1}^{2}+b_{1}^{2}}$

$e=2 \sqrt{\frac{a_{2}^{2}+b_{2}^{2}}{a_{1}^{2}+b_{1}^{2}}}$,

$\omega=\arctan \left(\frac{b_{2}\left(b_{1}^{2}-a_{1}^{2}\right)+2 a_{1} a_{2} b_{1}}{a_{2}\left(a_{1}^{2}-b_{1}^{2}\right)+2 a_{1} b_{1} b_{2}}\right)$.

Based on the above $\mathrm{O}-\mathrm{C}$ analysis, a quadratic term should be added to Eq. (15), which leads to:

$$
\begin{aligned}
\mathrm{O}-\mathrm{C}= & a_{0}+\alpha E+\beta E^{2}+a_{1} \cos (\Omega E)+b_{1} \sin (\Omega E) \\
& +a_{2} \cos (2 \Omega E)+b_{2} \sin (2 \Omega E),
\end{aligned}
$$

where $\alpha$ and $\beta$ are the coefficients of linear and quadratic terms, respectively. All best-fit coefficients are listed in Table 2 . We obtained $F(6,218)=16.2$, which corresponds to $a \sim 99.99 \%$ confidence level for a quadric-plus-eccentric fit. The mass function of the third component, $f\left(m_{3}\right)$, can be estimated by the formula

$f\left(m_{3}\right)=\frac{4 \pi^{2}}{G P_{3}^{2}} \times(a \sin (i))^{3}$.

Since the eccentricity $e=0.12$ is near zero, the elliptic orbit should be close to the circular orbit. Accordingly, the parameters of the third body derived from an eccentric fit are similar to that derived from a strictly sinusoidal fit (see Table 3 ). In addition, the $\chi^{2}$ values for the eccentric fit and strictly sinusoidal fit are nearly the same. This means that the elliptic orbit of the third body with eccentricity $e=0.12$ also cannot explain the departure near 60000 cycles in the $\mathrm{O}-\mathrm{C}$ diagram. Therefore, this episode may imply a sudden change in the orbital period of DQ Herculis caused by an unknown mechanism. Assuming a combined mass of $0.6( \pm 0.07) M_{\odot}+0.4( \pm 0.05) M_{\odot}$ for the eclipsing pair of DQ Herculis (Horne et al. 1993), the relationship between the mass of the third body, $M_{3}$, and its orbital inclination, $i$, is displayed in the left panel of Fig. 7. The third body in DQ Herculis could be a brown dwarf, as long as $i>20^{\circ}$, which implies that it is a

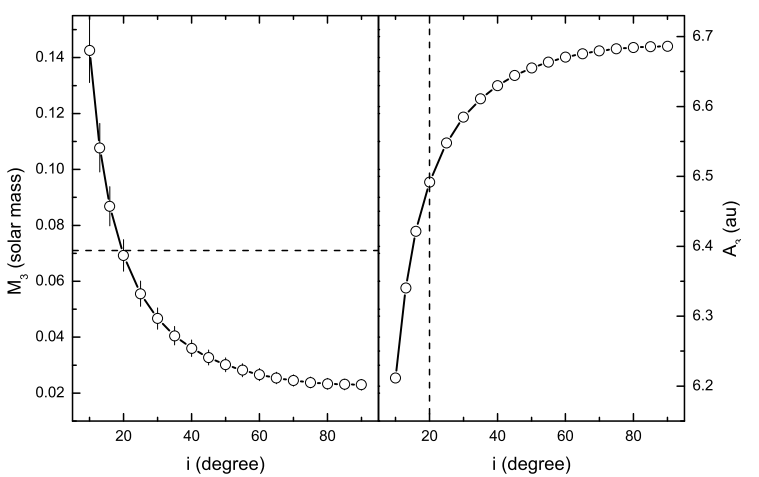

Fig. 7. The relationship between the mass of the third body and its orbital inclination in the left panel, and the distances between the third body and mass center corresponding to different inclinations are in the right panel. The dashed line in the left and right panels denotes the upper limit of the masses of the brown dwarf $\left(\sim 0.071 M_{\odot}\right)$, and $i=20^{\circ}$, respectively.

Table 3. The parameters of the elliptic and circular orbit

\begin{tabular}{ccc}
\hline \hline Parameters & Circular orbit & Elliptical orbit \\
\hline$\omega$ & & $-1.4(3)$ \\
$\mathrm{e}$ & 0 & $0.12(6)$ \\
$P_{3}$ (yr) & $17.7(3)$ & $17.9(3)$ \\
$a \sin i$ (A.U.) & $0.154(3)$ & $0.159(3)$ \\
$f\left(m_{3}\right)\left(M_{\odot}\right)$ & $0.0000116(8)$ & $0.0000125(8)$ \\
\hline$\sigma$ & $1.17 \times 10^{-3}$ & $1.18 \times 10^{-3}$ \\
\hline$\chi^{2}$ & 36.4 & 36.8 \\
\hline
\end{tabular}

brown dwarf with a confidence level $\gtrsim 77.8 \%$ based on the inclination, and its distance from the binary system shown in the right panel of Fig. 7 is $\sim 6.49( \pm 0.95)$ au, nearly $10^{3}$ times the binary separation. Since the red dwarf of DQ Herculis should be a low mass main sequence star, it cannot expand too much to swallow the remote third body when it reaches the red giant stage. This indicates that the brown dwarf could have survived the common envelope evolution of the parent binary. Assuming an orbit of the third body almost coplanar to the nova DQ Herculis with inclination $i \approx 86.5^{\circ}$ (Horne et al. 1993), the third body may be a massive extrasolar planet with a mass of $M_{3} \simeq 0.02 M_{\odot}$, which is $\sim 20$ times larger than that of Jupiter.

\section{Conclusion}

All 226 available times of light minimum covering 54 years are compiled and a new O-C diagram of DQ Herculis is obtained. The fit shown in Fig. 5 implies that the best-fit of the $\mathrm{O}-\mathrm{C}$ diagram is a sinusoidal variation with an $\sim 17.7( \pm 0.3) \mathrm{yr}$ period superimposed on the secular orbital period increase. This resolves the long-term debate of whether the orbital period of DQ Herculis is increasing or not. The increased orbital period is calculated to be $9.5( \pm 0.1) \times 10^{-12} \mathrm{~s} \mathrm{~s}^{-1}$. Using the absolute parameters of DQ Herculis derived by Horne et al. (1993), we estimated the lower limit of the mass transfer rate to be $7.2( \pm 3.2) \times 10^{-9} M_{\odot} \mathrm{yr}^{-1}$. In order to check the results of the orbital period increase presented in this paper, photometric observations with a longer base line are needed.

Although there is a small departure near 60000 cycles, the overall trend in the $\mathrm{O}-\mathrm{C}$ diagram can be well described by a quadric-plus-sinusoidal formula. Since there is no detailed analysis for the episodes of quasi-period oscillation, we investigated three plausible mechanisms, which are a red dwarf radius variation, Applegate's mechanism and the light travel-time effect, to 
explain the observed orbital period variation. According to the first mechanism, we concluded that the red dwarf's radius variation with an amplitude $A \sim 1.43( \pm 0.03) \times 10^{-6}$ is sufficient to account for the small quasi-sinusoidal oscillation seen in Fig. 5. But this requires too much energy, and the time scale $t_{\mathrm{ch}}$ is far longer than the oscillation period. Thus a direct change in radius of the tidally distorted secondary star cannot explain the observed quasi-periodic variation. Then Applegate's mechanism was used to probe whether the solar-type magnetic activity is responsible for the observed modulations in the $\mathrm{O}-\mathrm{C}$ diagram. However, this still gives a negative result. In order to show that the magnetic activity cycle of the red dwarf star in DQ Herculis is strong enough to cause the observed modulation in the $\mathrm{O}-\mathrm{C}$ diagram, A detailed analysis of variation in its brightness is necessary. Finally, an eccentric orbit of the tertiary component with a small eccentricity was attempted to explain the small departure displayed in the top panel of Fig. 5. The derived orbital eccentricity, $e=0.12$, is small enough, which means that the parameters of the third body derived from an elliptic orbit are similar to that derived from the circular orbit (see Table 3 ). This suggests that further studies are needed to explain the departure occurring in $~ 1985$. Based on the analysis of the light travel-time effect, a brown dwarf as a third component of the system is possible, with a significance level of $\gtrsim 77.8 \%$. Alternatively, the third body may be a massive extrasolar planet, as long as the orbital inclination of the third body is high enough. However, more convincing evidence, for example, further studies on the behavior of the $71 \mathrm{~s}$ oscillation in DQ Herculis, are needed to confirm the results in this paper.

Acknowledgements. This work was partly Supported by Special Foundation of the President of the Chinese Academy of Sciences and West Light Foundation of The Chinese Academy of Sciences, Yunnan Natural Science Foundation (2008CD157), the Yunnan Natural Science Foundation (No. 2005A0059M) and Chinese Natural Science Foundation (No.10573032, No. 10573013 and No.10433030). CCD photometric observations of DQ Herculis were obtained with the 1.0-m telescope at Yunnan Observatory. We thank the referee very much for the helpful comments and suggestions that helped to improve this paper greatly.

\section{References}

Africano, J., \& Olson, E. 1981, PASP, 93, 130

Applegate, J. H. 1992, ApJ, 385, 621

Bianchini, A., Mastrantonio, E., Canterna, R., Stute, J., \& Cantrell, K. 2004, A\&A, 426, 669

Borkovits, T., \& Hegedues, T. 1996, A\&AS, 120, 63

Campbell, L. 1935, Harvard College Observatory Bulletin, 898, 20

Dibai, É. A., \& Shakhovskoi, N. M. 1966, SvA, 10, 1059

Diethelm, R. 2003, IBVS, 5438

Diethelm, R. 2004, IBVS, 5543

Hall, D. S. 1991, ApJ, 380, L85

Horne, K., Welsh, W. F., \& Wade, R. A. 1993, ApJ, 410, 357

Irwin, J. B. 1952, ApJ, 116, 211

Kopal, Z. 1959, Close Binary Systems (London: Chapman \& Hall)

Kopal, Z. 1978, Dynamics of Close Binary Systems, D. Reidel. Co.

Kraft, R. P. 1959, ApJ, 130, 110

Livio, M. 1992, in Interacting Binaries, 22nd SF Advanced Course, ed. H. Nussbaumer

Locher, K. 1978, BBSAG Bull., 39, 4

Locher, K. 1979, BBSAG Bull., 42, 2

Locher, K. 1979, BBSAG Bull., 43, 2

Locher, K. 1979, BBSAG Bull., 44, 3

Locher, K. 1979, BBSAG Bull., 45, 4

Locher, K. 1980, BBSAG Bull., 46, 3

Locher, K. 1980, BBSAG Bull., 47, 3

Locher, K. 1980, BBSAG Bull., 48, 2

Locher, K. 1980, BBSAG Bull., 49, 4

Locher, K. 1980, BBSAG Bull., 50, 3
Locher, K. 1981, BBSAG Bull., 54, 2 Locher, K. 1981, BBSAG Bull., 55, 2 Locher, K. 1981, BBSAG Bull., 56, 5 Locher, K. 1981, BBSAG Bull., 57, 4 Locher, K. 1982, BBSAG Bull., 58, 3 Locher, K. 1982, BBSAG Bull., 59, 2 Locher, K. 1982, BBSAG Bull., 60, 3 Locher, K. 1982, BBSAG Bull., 61, 3 Locher, K. 1982, BBSAG Bull., 63, 2 Locher, K. 1983, BBSAG Bull., 64, 4 Locher, K. 1983, BBSAG Bull., 65, 3 Locher, K. 1983, BBSAG Bull., 66, 3 Locher, K. 1983, BBSAG Bull., 67, 3 Locher, K. 1983, BBSAG Bull., 68, 4 Locher, K. 1983, BBSAG Bull., 69, 4 Locher, K. 1984, BBSAG Bull., 71, 2 Locher, K. 1984, BBSAG Bull., 72, 2 Locher, K. 1984, BBSAG Bull., 73, 4 Locher, K. 1984, BBSAG Bull., 84, 3 Locher, K. 1985, BBSAG Bull., 77, 4 Locher, K. 1986, BBSAG Bull., 80, 3 Locher, K. 1986, BBSAG Bull., 81, 3 Locher, K. 1986, BBSAG Bull., 82, 4 Locher, K. 1987, BBSAG Bull., 83, 3 Locher, K. 1987, BBSAG Bull., 84, 3 Locher, K. 1987, BBSAG Bull., 85, 3 Locher, K. 1988, BBSAG Bull., 88, 4 Locher, K. 1988, BBSAG Bull., 89, 7 Locher, K. 1989, BBSAG Bull., 92, 3 Locher, K. 1990, BBSAG Bull., 94, 3 Locher, K. 1990, BBSAG Bull., 95, 3 Locher, K. 1990, BBSAG Bull., 96, 5 Locher, K. 1991, BBSAG Bull., 97, 6 Locher, K. 1991, BBSAG Bull., 98, 7 Locher, K. 1993, BBSAG Bull., 104, 5 Locher, K. 1994, BBSAG Bull., 106, 5 Locher, K. 1994, BBSAG Bull., 107, 8 Locher, K. 1995, BBSAG Bull., 108, 7 Locher, K. 1995, BBSAG Bull., 109, 9 Locher, K. 1996, BBSAG Bull., 112, 7 Locher, K. 1997, BBSAG Bull., 114, 8 Locher, K. 1998, BBSAG Bull., 117, 8 Locher, K. 1998, BBSAG Bull., 118, 6 Locher, K. 2000, BBSAG Bull., 121, 6 Locher, K. 2001, BBSAG Bull., 126, 5 Locher, K. 2002, BBSAG Bull., 127, 5

Marsh, T. R., \& Pringle, J. E. 1990, ApJ, 365, 677

Martin, P. G. 1989, in Classical Novae, ed. M. F. Bode, \& A. Evans (Chichester: Wiley), Chap. 6

Mumford, G. S. 1969, in Mass Loss from Stars, ed. M. Hack (Dordrecht: Reidel Publishing Co.), 204

Nagai, K. 2006, Var. Star. Bull. Japan, 44

Nather, R. E., \& Warner, B. 1969, MNRAS, 143, 145

Nelson, M. R., \& Olson, E. C. 1976, ApJ, 207, 195

Patterson, J. 1994, PASP, 106, 209

Patterson, J., Robinson, E. L., \& Nather, R. E. 1978, ApJ, 224, 570

Pringle, J. E. 1975, MNRAS, 170, 633

Robinson, E. L. 1976, ApJ, 203, 485

Schneider, D. P., \& Greenstein, J. L. 1979, ApJ, 233, 935

Schoembs, R., \& Rebhan, H. 1989, A\&A, 224, 42

Smak, J. 1971, Acta Astron., 21, 15

Smak, J. 1980, Acta Astron., 30, 267

Smak, J. 2004, Acta Astron., 54, 433

Starrfield, S, 1989, in Classical Novae, ed. M. F. Bode, \& A. Evans (Chichester: Wiley), Chap. 3

Swedlund, J. B., Kemp, J. C., \& Wolstencroft, R. D. 1974, ApJ, 193, 11

Vaytet, N. M. H., O’Brien, T. J., \& Rushton, A. P. 2007, MNRAS, 380, 175

Walker, M. F. 1956, ApJ, 123, 68

Walker, M. F. 1958, ApJ, 127, 319

Warner, B. 1988, Nature 336, 10

Warner, B., \& Nather, R. E. 1971, MNRAS, 152, 219

Warner, B., Peters, W. L., Hubbard, W. B, \& Nather, R. E. 1972, MNRAS, 159, 321

Wood, M. A., Robertson, J. R., \& Simpson, J. C., et al. 2005, ApJ, 634, 570

Young, P. J., Schneider, D. P. 1981, ApJ, 247, 960

Zhang, E., Robinson, E. L., Stiening, R. F., \& Horne, K. 1995, ApJ, 454, 447 
Z. B. Dai and S. B. Qian: Plausible explanations for the variations of orbital period in the old nova DQ Herculis, Online Material $p 1$

Table 1. The times of light minimum for the old nova DQ Herculis.

\begin{tabular}{|c|c|c|c|c|c|c|c|c|c|c|c|c|}
\hline $\begin{array}{l}\text { JD. Hel. } \\
2400000+\end{array}$ & Type Error & method & $E$ (cycle) & $(\mathrm{O}-\mathrm{C})^{d}$ & Ref. & $\begin{array}{l}\text { JD. Hel. } \\
2400000+\end{array}$ & Type & Error & Method & $E$ (cycle) & 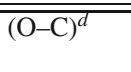 & Ref. \\
\hline 34954.751500 & pri & pe & -1 & 0.00204 & (1) & 42189.974640 & pri & & pe & 37367 & -0.00139 & (6) \\
\hline 34954.944950 & pri & pe & 0 & 0.00184 & (1) & 42191.910790 & pri & & pe & 37377 & -0.00150 & (6) \\
\hline 34955.719060 & pri & pe & & & (2) & 42193.072620 & pri & & pe & 37383 & -0.00140 & (6) \\
\hline 34955.719100 & pri & pe & & & (3) & 42223.665520 & pri & 0.00068 & pe & 37541 & -0.00057 & (3) \\
\hline $34955.718940^{c r}$ & pri & pe & 4 & 0.00139 & (1) & 42245.737350 & pri & & pe & 37655 & -0.00150 & (3) \\
\hline 34981.665000 & pri & pg & 138 & 0.00223 & (1) & 42329.576020 & pri & & pe & 38088 & -0.00071 & (3) \\
\hline 34983.794960 & pri & pe & 149 & 0.00233 & (1) & 42342.548590 & pri & & pe & 38155 & -0.00075 & (3) \\
\hline 34984.762980 & pri & pe & 154 & 0.00233 & (1) & 42513.903900 & pri & & pe & 39040 & 0.00004 & (3) \\
\hline 34985.729820 & pri & pe & 159 & 0.00103 & (1) & 42514.872200 & pri & & pe & 39045 & 0.00024 & (3) \\
\hline 34989.797000 & pri & pg & 180 & 0.00213 & (1) & 42515.840500 & pri & & pe & 39050 & 0.00043 & (3) \\
\hline 35218.851030 & pri & pe & 1363 & 0.00260 & (2) & 42569.858820 & pri & & pe & 39329 & -0.00144 & (3) \\
\hline 35243.827480 & pri & pe & 1492 & 0.00200 & (2) & 42571.794290 & pri & & pe & 39339 & -0.00224 & (3) \\
\hline 35374.715180 & pri & pe & 2168 & 0.00198 & (2) & 42593.675500 & pri & & pe & 39452 & -0.00017 & (3) \\
\hline 35659.917250 & pri & pe & 3641 & 0.00040 & (2) & 42605.679900 & pri & & pe & 39514 & -0.00027 & (3) \\
\hline 35660.691900 & pri & pe & 3645 & 0.00057 & (2) & 42606.841700 & pri & & pe & 39520 & -0.00020 & (3) \\
\hline 35660.884980 & pri & pe & 3646 & 0.00003 & (2) & 42662.602780 & pri & & pe & 39808 & -0.00196 & (3) \\
\hline 35695.736950 & pri & pe & 3826 & 0.00024 & (2) & 42925.928210 & pri & & pe & 41168 & -0.00097 & (3) \\
\hline 35695.930620 & pri & pe & 3827 & 0.00029 & (2) & 42926.896650 & pri & & pe & 41173 & -0.00063 & (3) \\
\hline 35696.705600 & pri & pe & 3831 & 0.00078 & (2) & 42927.865260 & pri & & pe & 41178 & -0.00013 & (3) \\
\hline 35697.673600 & pri & pe & 3836 & 0.00068 & (2) & 42928.833320 & pri & & pe & 41183 & -0.00017 & (3) \\
\hline 35697.867250 & pri & pe & 3837 & 0.00071 & (2) & 42929.801430 & pri & & pe & 41188 & -0.00017 & (3) \\
\hline 36103.696500 & pri & pe & 5933 & 0.00051 & (3) & 42934.835800 & pri & & pe & 41214 & 0.00006 & (3) \\
\hline 36351.918500 & pri & pe & 7215 & 0.00048 & (3) & 42935.803800 & pri & & pe & 41219 & -0.00004 & (3) \\
\hline 36376.894800 & pri & pe & 7344 & -0.00032 & (3) & 42955.747400 & pri & & pe & 41322 & 0.00060 & (3) \\
\hline 36393.740200 & pri & pe & 7431 & 0.00006 & (3) & 42991.760200 & pri & & pe & 41508 & -0.00009 & (3) \\
\hline 36399.742400 & pri & pe & 7462 & 0.00002 & (3) & 42993.696900 & pri & & pe & 41518 & 0.00040 & (3) \\
\hline 36407.874300 & pri & pe & 7504 & -0.00016 & (3) & 42994.665000 & pri & & pe & 41523 & 0.00039 & (3) \\
\hline 36487.646200 & pri & pe & 7916 & -0.00008 & (3) & 43007.637100 & pri & & pe & 41590 & -0.00011 & (3) \\
\hline 36785.822800 & pri & pe & 9456 & 0.00030 & (3) & 43310.847760 & pri & & pe & 43156 & 0.00019 & (3) \\
\hline 39060.288600 & pri & pe & 21203 & 0.00113 & (4) & 43716.870520 & pri & 0.00015 & pe & 45253 & -0.00012 & (3) \\
\hline 40006.900700 & pri & pe & 26092 & 0.00055 & (3) & 43808.261000 & pri & & pv & 45725 & 0.00130 & (7) \\
\hline 40007.868600 & pri & pe & 26097 & 0.00034 & (3) & 43913.008800 & pri & & pe & 46266 & 0.00016 & (3) \\
\hline 40070.795600 & pri & pe & 26422 & 0.00054 & (3) & 43940.505000 & pri & & pv & 46408 & 0.00218 & (8) \\
\hline 40073.699700 & pri & pe & 26437 & 0.00033 & (3) & 43942.634000 & pri & & pv & 46419 & 0.00138 & (8) \\
\hline 40073.892800 & pri & pe & 26438 & -0.00019 & (3) & 44024.531000 & pri & & pe & 46842 & -0.00333 & (9) \\
\hline 40074.668100 & pri & pe & 26442 & 0.00062 & (3) & 44054.739600 & pri & & pe & 46998 & 0.00045 & (3) \\
\hline 40074.861700 & pri & pe & 26443 & 0.00060 & (3) & 44073.525000 & pri & & pv & 47095 & 0.00466 & (10) \\
\hline 40417.763000 & pri & pe & 28214 & -0.00076 & (3) & 44079.525000 & pri & & pv & 47126 & 0.00236 & (10) \\
\hline 40418.925500 & pri & pe & 28220 & 0.00002 & (3) & 44082.429000 & pri & & pv & 47141 & 0.00206 & (10) \\
\hline 40441.772000 & pri & pe & 28338 & -0.00075 & (3) & 44100.819670 & pri & & pe & 47236 & -0.00126 & (3) \\
\hline 40443.901500 & pri & pe & 28349 & -0.00107 & (3) & 44105.662400 & pri & & pe & 47261 & 0.00096 & (3) \\
\hline 40444.676500 & pri & pe & 28353 & -0.00056 & (3) & 44110.695800 & pri & & pe & 47287 & 0.00020 & (3) \\
\hline 40444.870500 & pri & pe & 28354 & -0.00018 & (3) & 44111.665000 & pri & & pe & 47292 & 0.00126 & (3) \\
\hline 40445.645000 & pri & pe & 28358 & -0.00017 & (3) & 44111.857400 & pri & & pe & 47293 & 0.00008 & (3) \\
\hline 40445.838500 & pri & pe & 28359 & -0.00029 & (5) & 44139.738200 & pri & & pe & 47437 & -0.00054 & (3) \\
\hline 40446.806000 & pri & pe & 28364 & -0.00089 & (3) & 44140.706900 & pri & & pe & 47442 & 0.00005 & (3) \\
\hline 41215.675000 & pri & pe & 32335 & -0.00057 & (3) & 44142.643800 & pri & & pe & 47452 & 0.00075 & (3) \\
\hline 41220.709000 & pri & pe & 32361 & -0.00072 & (3) & 44143.420000 & pri & & pv & 47456 & 0.00245 & (11) \\
\hline 41917.743940 & pri & pe & 35961 & -0.00110 & (3) & 44267.723000 & pri & & pv & 48098 & 0.00084 & (12) \\
\hline 41918.712160 & pri & pe & 35966 & -0.00098 & (3) & 44289.602000 & pri & & pv & 48211 & 0.00064 & (12) \\
\hline 41945.625760 & pri & pe & 36105 & -0.00069 & (3) & 44341.493000 & pri & & pv & 48479 & 0.00123 & (13) \\
\hline 41948.723150 & pri & pe & 36121 & -0.00126 & (3) & 44343.428000 & pri & & pv & 48489 & 0.00006 & (13) \\
\hline 41979.703400 & pri & pe & 36281 & -0.00033 & (3) & 44370.537000 & pri & & pv & 48629 & 0.00212 & (14) \\
\hline 41980.671000 & pri & pe & 36286 & -0.00084 & (3) & 44396.482000 & pri & & pv & 48763 & 0.00192 & (14) \\
\hline 41981.639400 & pri & pe & 36291 & -0.00054 & (3) & 44456.507000 & pri & & pv & 49073 & 0.00441 & (15) \\
\hline 42130.144420 & pri & pe & 37058 & -0.00279 & (6) & 44461.538000 & pri & & pv & 49099 & 0.00131 & (15) \\
\hline 42131.112510 & pri & pe & 37063 & -0.00279 & (6) & 44466.573000 & pri & & pv & 49125 & 0.00211 & (15) \\
\hline 42132.080600 & pri & pe & 37068 & -0.00279 & (6) & 44487.483000 & pri & & pv & 49233 & 0.00111 & (16) \\
\hline 42161.124630 & pri & pe & 37218 & -0.00189 & (6) & 44704.532000 & pri & & pv & 50354 & 0.00108 & (17) \\
\hline 42162.092710 & pri & pe & 37223 & -0.00189 & (6) & 44770.556000 & pri & & pv & 50695 & 0.00030 & (18) \\
\hline 42163.060790 & pri & pe & 37228 & -0.00199 & (6) & 44809.474500 & pri & & pv & 50896 & 0.00097 & (19) \\
\hline 42164.028880 & pri & pe & 37233 & -0.00199 & (6) & 44812.572000 & pri & & pv & 50912 & 0.00057 & (19) \\
\hline 42164.996960 & pri & pe & 37238 & -0.00199 & (6) & 44819.542000 & pri & & pv & 50948 & 0.00021 & (19) \\
\hline 44848.392000 & pri & pv & 51097 & 0.00066 & (19) & 46649.452000 & pri & & pv & 60399 & -0.00113 & (40) \\
\hline 44853.428000 & pri & pv & & & (19) & 46651.580000 & pri & & pv & 60410 & -0.00297 & (41) \\
\hline 44853.429000 & pri & pv & & & (19) & 46907.549000 & pri & & pv & 61732 & -0.00082 & (42) \\
\hline $44853.428500^{c r}$ & pri & pv & 51123 & 0.00306 & (19) & 46952.469000 & pri & & pv & 61964 & -0.00087 & (43) \\
\hline 44885.374000 & pri & pv & 51288 & 0.00106 & (20) & 47037.468000 & pri & & pv & 62403 & -0.00146 & (44) \\
\hline
\end{tabular}


Table 1. continued.

\begin{tabular}{|c|c|c|c|c|c|c|c|c|c|c|c|c|c|}
\hline $\begin{array}{l}\text { JD. Hel. } \\
2400000+\end{array}$ & Type & Error & Method & $E$ (cycle) & $(\mathrm{O}-\mathrm{C})^{d}$ & Ref. & $\begin{array}{l}\text { JD. Hel. } \\
2400000+\end{array}$ & Type & Error & Method & $E$ (cycle) & $\overline{(\mathrm{O}-\mathrm{C})^{d}}$ & Ref. \\
\hline-44895.249000 & pri & & pv & 51339 & 0.00146 & $(21)$ & 47304.472000 & pri & & $\mathrm{pv}$ & 63782 & -0.00070 & $(45)$ \\
\hline 44910.351000 & pri & & pv & 51417 & 0.00096 & (20) & 47383.467000 & pri & & pv & 64190 & -0.00306 & (46) \\
\hline 45053.631000 & pri & & $\mathrm{pv}$ & 52157 & 0.00154 & (22) & 47746.507000 & pri & & pv & 66065 & -0.00231 & (47) \\
\hline 45101.456000 & pri & & pv & 52404 & 0.00213 & $(23,24)$ & 47942.643000 & pri & & pv & 67078 & -0.00423 & (48) \\
\hline 45140.373000 & pri & & pv & 52605 & 0.00133 & (24) & 48039.457000 & pri & & pv & 67578 & -0.00073 & (49) \\
\hline $45142.890400^{+}$ & pri & & pe & 52618 & 0.00163 & (25) & 48123.488000 & pri & & pv & 68012 & -0.00121 & (50) \\
\hline $45144.826400^{+}$ & pri & & pe & 52628 & 0.00143 & (25) & 48306.653000 & pri & 0.001 & pv & 68958 & -0.00160 & (51) \\
\hline $45203.686700^{+}$ & pri & & pe & 52932 & 0.00102 & (25) & $48384.875600^{+}$ & pri & & pe & 69362 & -0.00185 & (25) \\
\hline $45233.697600^{+}$ & pri & & pe & 53087 & 0.00061 & (25) & $48385.844000^{+}$ & pri & & pe & 69367 & -0.00155 & (25) \\
\hline $45234.666000^{+}$ & pri & & pe & 53092 & 0.00091 & (25) & 48390.492000 & pri & 0.001 & pv & 69391 & -0.00045 & (52) \\
\hline 45257.321000 & pri & & pv & 53209 & 0.00231 & (26) & $48776.765600^{+}$ & pri & & pe & 71386 & -0.00059 & (25) \\
\hline 45356.647000 & pri & & pv & 53722 & 0.00080 & (27) & 49158.393000 & pri & 0.001 & pv & 73357 & 0.00001 & (53) \\
\hline 45404.470000 & pri & & pv & 53969 & -0.00060 & (28) & 49475.546000 & pri & 0.003 & pv & 74995 & 0.00187 & (54) \\
\hline 45404.666000 & pri & & $\mathrm{pv}$ & & & (28) & $49476.898900^{+}$ & pri & & pe & 75002 & -0.00055 & (25) \\
\hline 45404.667000 & pri & & pv & & & (28) & $49477.866400^{+}$ & pri & & pe & 75007 & -0.00115 & (25) \\
\hline $45404.666500^{c r}$ & pri & & pv & 53970 & 0.00229 & (28) & 49568.481000 & pri & 0.002 & pv & 75475 & -0.00114 & (55) \\
\hline 45459.460000 & pri & & pv & 54253 & 0.00108 & (29) & 49799.474000 & pri & 0.002 & pv & 76668 & 0.00213 & (56) \\
\hline $45499.732200^{+}$ & pri & & pe & 54461 & 0.00011 & (25) & 49836.453000 & pri & 0.002 & pv & 76859 & -0.00050 & (57) \\
\hline $45499.925600^{+}$ & pri & & pe & 54462 & -0.00011 & (25) & 50278.489000 & pri & 0.001 & pe & 79142 & -0.00106 & (58) \\
\hline $45500.700400^{+}$ & pri & & pe & 54466 & 0.00020 & (25) & 50539.491000 & pri & 0.001 & pv & 80490 & -0.00007 & (59) \\
\hline $45500.893200^{+}$ & pri & & pe & 54467 & -0.00062 & (25) & $50630.686760^{+}$ & pri & & pe & 80961 & 0.00022 & (60) \\
\hline $45501.862200^{+}$ & pri & & pe & 54472 & 0.00028 & (25) & $50630.881350^{+}$ & pri & & pe & 80962 & 0.00122 & (60) \\
\hline $45502.829900^{+}$ & pri & & pe & 54477 & -0.00013 & (25) & $50631.655040^{+}$ & pri & & pe & 80966 & 0.00042 & (60) \\
\hline $45503.798000^{+}$ & pri & & pe & 54482 & -0.00013 & (25) & $50631.848880^{+}$ & pri & & pe & 80967 & 0.00062 & (60) \\
\hline $45506.896000^{+}$ & pri & & pe & 54498 & -0.00006 & (25) & 50632.430600 & pri & 0.0003 & pe & 80970 & 0.00152 & (61) \\
\hline 45547.557000 & pri & & pv & 54708 & 0.00057 & (30) & $50633.397620^{+}$ & pri & & pe & 80975 & 0.00042 & (60) \\
\hline 45548.330500 & pri & & pv & 54712 & -0.00045 & (31) & $50633.785110^{+}$ & pri & & pe & 80977 & 0.00062 & (60) \\
\hline 45564.403000 & pri & & pv & 54795 & 0.00147 & (31) & $50634.172100^{+}$ & pri & & pe & 80979 & 0.00042 & (60) \\
\hline 45610.290000 & pri & & pv & 55032 & 0.00037 & (32) & $50634.753100^{+}$ & pri & & pe & 80982 & 0.00052 & (60) \\
\hline 45621.326000 & pri & & pv & & & (32) & $50635.140380^{+}$ & pri & & pe & 80984 & 0.00062 & (60) \\
\hline 45621.327000 & pri & & $\mathrm{pv}$ & & & (32) & $50636.302000^{+}$ & pri & & pe & 80990 & 0.00052 & (60) \\
\hline $45621.326500^{c r}$ & pri & & pv & 55089 & 0.00046 & (32) & $50636.495800^{+}$ & pri & & pe & 80991 & 0.00062 & (60) \\
\hline 45701.674000 & pri & & pv & 55504 & -0.00475 & (33) & $50637.270320^{+}$ & pri & & pe & & & (60) \\
\hline 45753.569000 & pri & & pv & 55772 & -0.00012 & (34) & $50637.270380^{+}$ & pri & & pe & & & (60) \\
\hline 45766.538000 & pri & & pv & & & (34) & $50637.270350^{c r}$ & pri & & pe & 80995 & 0.00072 & (60) \\
\hline 45766.540000 & pri & & pv & & & (34) & $50637.463720^{+}$ & pri & & pe & 80996 & 0.00042 & (60) \\
\hline 45766.541000 & pri & & pv & & & (34) & $50638.238300^{+}$ & pri & & pe & 81000 & 0.00052 & (60) \\
\hline 45766.541000 & pri & & pv & & & (34) & $50639.206300^{+}$ & pri & & pe & 81005 & 0.00042 & (60) \\
\hline 45766.543000 & pri & & pv & & & (34) & 50925.570000 & pri & 0.002 & pv & 824 & -0.00119 & (62) \\
\hline 45766.543000 & pri & & pv & & & (34) & 50954.421000 & pri & 0.002 & pv & 82633 & 0.00028 & (63) \\
\hline $45766.541000^{c r}$ & pri & & pv & 55839 & -0.00072 & (34) & 51458.416000 & pri & 0.001 & pv & 85236 & 0.00001 & (64) \\
\hline 45868.387000 & pri & & pv & 56365 & 0.00063 & (35) & 52202.309000 & pri & 0.001 & pv & $890^{\top}$ & 0.00142 & (65) \\
\hline 45905.560000 & pri & & pv & 56557 & -0.00154 & (36) & 52348.491000 & pri & 0.002 & pv & 89833 & -0.00030 & (66) \\
\hline 45906.528000 & pri & & pv & 56562 & -0.00165 & (36) & 52413.548200 & pri & 0.0005 & pe & 90169 & 0.00019 & (67) \\
\hline 46256.400000 & pri & & pv & 58369 & -0.00262 & (37) & 52763.421000 & pri & 0.001 & pv & 91976 & 0.00005 & (68) \\
\hline 46590.397000 & pri & & pv & 60094 & -0.00174 & (38) & $52809.698340^{+}$ & pri & & pe & 92215 & 0.00194 & (60) \\
\hline 46622.344000 & pri & & pv & & & (38) & $52809.892000^{+}$ & pri & & pe & 92216 & 0.00204 & (60) \\
\hline 46622.345000 & pri & & pv & & & (38) & $52810.666560^{+}$ & pri & & pe & 92220 & 0.00204 & (60) \\
\hline $46622.344500^{c r}$ & pri & & pv & 60259 & -0.00169 & (38) & $52810.860280^{+}$ & pri & & pe & 92221 & 0.00214 & (60) \\
\hline $46626.603700^{+}$ & pri & 0.0005 & pe & & & (25) & $52815.700700^{+}$ & pri & & pe & 92246 & 0.00204 & (60) \\
\hline 46626.603100 & pri & 0.0005 & pe & 60281 & -0.00277 & (39) & $52815.894410^{+}$ & pri & & pe & 92247 & 0.00214 & (60) \\
\hline $46627.571400^{+}$ & pri & 0.0004 & pe & & & (25) & 53149.502000 & pri & 0.001 & pv & 93970 & 0.00090 & (69) \\
\hline 46627.570800 & pri & 0.0004 & pe & 60286 & -0.00317 & (39) & 53475.173100 & pri & & $\mathrm{ced}$ & 95652 & 0.00165 & (70) \\
\hline 46648.483000 & pri & & pv & & & (40) & $54507.364570^{N}$ & pri & 0.0001 & $\mathrm{ced}$ & 100983 & -0.00008 & (71) \\
\hline 46648.485000 & pri & & pv & & & $(+)$ & $54554.415230^{R}$ & pri & 0.00015 & $\mathrm{ccd}$ & 101226 & 0.00072 & (71) \\
\hline $46648.484000^{c r}$ & pri & & pv & 60394 & -0.00102 & (40) & & & & & & & \\
\hline
\end{tabular}

Note. ${ }^{+}$HJED data. ${ }^{c r}$ the corrected data used in this paper, which corresponds to the above data without cycles and O-C. ${ }^{N}$ no filter used in observation. ${ }^{R}$ the $R$-filter used in observation. References: (1) Swedlund et al. (1974); (2) Vaytet et al. (2007); (3) Africano \& Olson (1981); (4) Dibai \& Shakhovskoi (1966); (5) Walker (1958); (6) Smak (2004); (7) BBSAG Bull. 39 (1978); (8) BBSAG Bull. 42 (1979); (9) BBSAG Bull. 43 (1978); (10) BBSAG Bull. 44 (1978); (11) BBSAG Bull. 45 (1979); (12) BBSAG Bull. 46 (1980); (13) BBSAG Bull. 47 (1980); (14) BBSAG Bull. 48 (1980); (15) BBSAG Bull. 49 (1980); (16) BBSAG Bull. 50 (1980); (17) BBSAG Bull. 54 (1981); (18) BBSAG Bull. 55 (1981); (19) BBSAG Bull. 56 (1981); (20) BBSAG Bull. 57 (1981); (21) BBSAG Bull. 58 (1982); (22) BBSAG Bull. 59 (1982); (23) BBSAG Bull. 60 (1982); (24) BBSAG Bull. 61 (1982); (25) Warner (1988); (26) BBSAG Bull. 63 (1982); (27) BBSAG Bull. 64 (1983); (28) BBSAG Bull. 65 (1983); (29) BBSAG Bull. 66 (1983); (30) BBSAG Bull. 67 (1983); (31) BBSAG Bull. 68 (1983); (32) BBSAG Bull. 69 (1983); (33) BBSAG Bull. 70 (1984); (34) BBSAG Bull. 71 (1984); (35) BBSAG Bull. 72 (1984); (36) BBSAG Bull. 73 (1984); (37) BBSAG Bull. 77 (1985); (38) BBSAG Bull. 80 (1986); (39) Robinson (1976); (40) BBSAG Bull. 82 (1986); (41) BBSAG Bull. 81 (1986); (42) BBSAG Bull. 83 (1987); (43) BBSAG Bull. 84 (1987); (44) BBSAG Bull. 85 (1987); (45) BBSAG Bull. 88 (1988); (46) BBSAG Bull. 89 (1988); (47) BBSAG Bull. 92 (1989); (48) BBSAG Bull. 94 (1990); (49) BBSAG Bull. 95 (1990); (50) BBSAG Bull. 96 (1990); (51) BBSAG Bull. 97 (1991); (52) BBSAG Bull. 98 (1991); (53) BBSAG Bull. 104 (1993); (54) BBSAG Bull. 106 (1994); (55) BBSAG Bull. 107 (1994); (56) BBSAG Bull. 108 (1995); (57) BBSAG Bull. 109 (1995); (58) BBSAG Bull. 112 (1996); (59) BBSAG Bull. 114 (1997); (60) Wood et al. (2005); (61) Krzesinski unpublished (1999); (62) BBSAG Bull. 117 (1998); (63) BBSAG Bull. 118 (1998); (64) BBSAG Bull. 121 (2000); (65) BBSAG Bull. 126 (2001); (66) BBSAG Bull. 127 (2002); (67) Bianchini et al. (2004); (68) Diethelm (2003); (69) Diethelm (2004); (70) Nagai (2006); (71) This paper. 